\title{
Organic Polymer Solar Cell Morphology Characterization with AFM, TEM and Helium Ion Microscopy (HIM)
}

Emile van Veldhoven*, Hans van der Veer*, Diederik Maas*, Peter Graat**, Marcel A. Verheijen**, Yulia Galagan*** and Ronn Andriessen***

* TNO Science and Industry - Van Leeuwenhoek laboratory, P.O. Box 155, 2600AD Delft, The Netherlands

** Philips Research, High Tech Campus 11, 5656AE Eindhoven, the Netherlands.

*** Holst Centre, PO Box 8550, 5605 KN Eindhoven, the Netherlands

To obtain efficient bulk hetero-junction polymer solar cells, the scale of phase separation should be closely related to the exciton diffusion lengths in the two materials involved. The morphology can be controlled by many processing parameters during the production and does influence the final cell efficiency [1].

To optimize the production process it is important to have a high resolution imaging tool that is capable of characterizing layers in the device. However, the sample-probe interaction may cause artifacts to the images of the sample. Until recently, the imaging tools have been limited toward AFM and TEM. Helium Ion Microscopy (HIM) is a relative new high resolution surface imaging technique [2]. To study the feasibility of HIM for imaging these materials a comparison study has been performed. Samples containing a mixture of polymers and fullerenes have been prepared in various ways to obtain variations in morphology. All samples have been studied using HIM, AFM and TEM. The challenge for this class of samples is in obtaining high-resolution images of a nonconductive layer without the creation of artifacts due to sample preparation, charging, or due to contaminating or damaging the surface. In this contribution we will discuss the obtained results.

TEM has a resolution below $1 \mathrm{~nm}$, but plan-view studies of the organic photovoltaic (OPV) layer require sample preparation to remove underlying layers [3]. Depending on sample process conditions (e.g. thermal cycling) it appeared cumbersome to completely remove the underlying layers, yielding a superposition of information of the various layers, complicating interpretation of the images.

AFM has as major advantage that it can be used without any sample preparation and does not require a vacuum. However, the resolution might be limited by the $5-10 \mathrm{~nm}$ tip radius and thus prevent the observation in high contrast of the small clusters inside the polymer solar cells. Figure 1 shows a high resolution AFM phase image of an annealed solar cell. This image indicates how the polymers are oriented in the active layer.

HIM deploys a sub-nanometer sized spot that scans the surface of the sample with high sensitivity and high speed. The technique does not require complicated sample preparation. Sample charging is cancelled by usage of an electron flood gun that was synchronized with the line scan. Figure 2 shows an example of a high resolution image made by the HIM, showing many similarities with the AFM phase image. This makes HIM a new and very direct imaging technique for this class of samples.

[1] D. Veldman et al. JACS 130 ( 2008), 7721

[2] A.E. Vladár, M.T. Postek, and B. Ming, Microsc.Today, 3 (2009) 6-13

[3] S. van Bavel et al. Nano Letters 9 (2009) 507. 


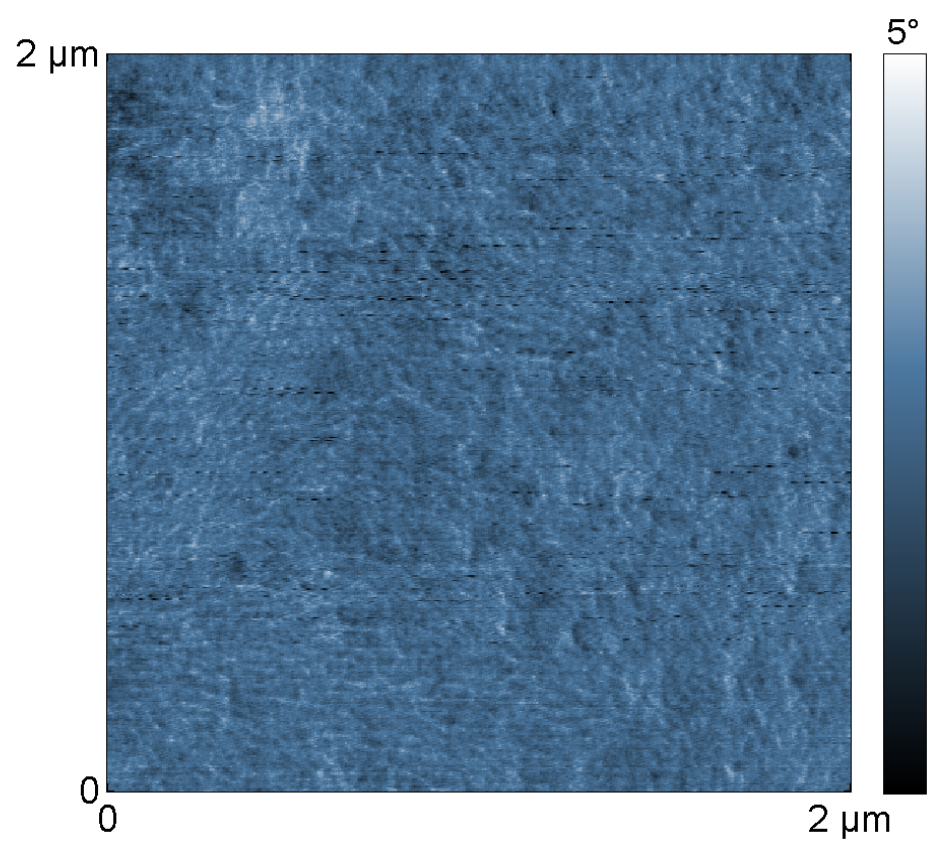

Figure 1: High resolution AFM phase image of the active layer of an annealed polymer solar cell. The phase contrast is an indication on how the polymers are oriented.

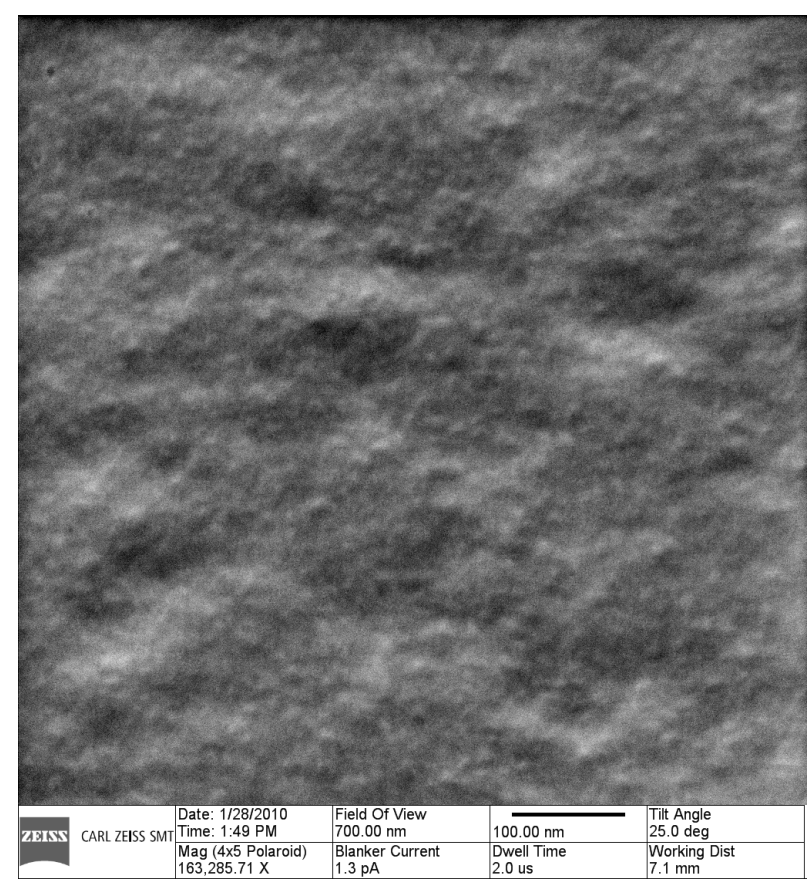

Figure 2: High resolution HIM image of the active layer of an annealed polymer solar cell. The relative large features of about $100 \mathrm{~nm}$ is most likely due to the surface roughness of the sample, the smaller features are an indication on how the polymers are oriented and formed clusters due to annealing process. 